\title{
Tailoring the vortex core in confined magnetic nanostructures
}

Thiago R. S. Moura, Felipe F. Oliveira, A. S. Carriço, Ana L. Dantas, and G. O. G. Rebouças

Citation: Journal of Applied Physics 111, $07 D 116$ (2012);

View online: https://doi.org/10.1063/1.3675989

View Table of Contents: http://aip.scitation.org/toc/jap/111/7

Published by the American Institute of Physics

\section{Articles you may be interested in}

Controlling the core-to-core distance of vortex pairs in exchange-biased iron elliptical nanoelements Journal of Applied Physics 111, 07D102 (2012); 10.1063/1.3670978

Nucleation of vortex pairs in exchange biased nanoelements

Journal of Applied Physics 109, 07D314 (2011); 10.1063/1.3537924

Controlling the vortex core of thin Permalloy nano-cylinders dipolar coupled to Co polarizers

Journal of Applied Physics 115, 17D110 (2014); 10.1063/1.4860056

Phase locking of vortex based spin transfer oscillators to a microwave current

Applied Physics Letters 98, 132506 (2011); 10.1063/1.3565159

\section{Scilight}

Sharp, quick summaries illuminating the latest physics research

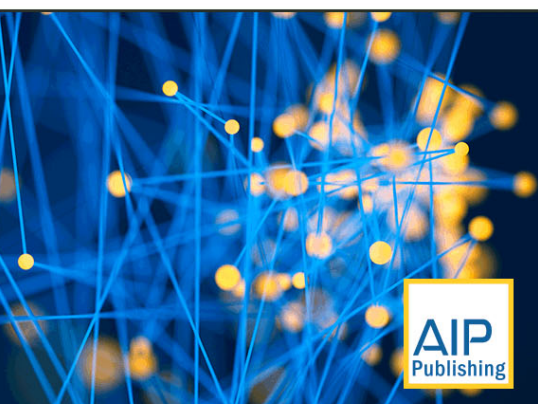




\title{
Tailoring the vortex core in confined magnetic nanostructures
}

\author{
Thiago R. S. Moura, ${ }^{1}$ Felipe F. Oliveira, ${ }^{1}$ A. S. Carriço, ${ }^{1, a)}$ Ana L. Dantas, ${ }^{2}$ \\ and G. O. G. Rebouças ${ }^{3}$ \\ ${ }_{1}^{1}$ Departamento de Física, Universidade Federal do Rio Grande do Norte, 59072-970—Natal—RN, Brazil \\ ${ }^{2}$ Departamento de Física, Universidade do Estado do Rio Grande do Norte, 59610-210_Mossoró-RN, Brazil \\ ${ }^{3}$ Departamento de Física, Universidade Federal do Semi-Arido, 59515-000-Angicos-RN, Brazil
}

(Presented 1 November 2011; received 23 September 2011; accepted 8 November 2011; published online 2 March 2012)

\begin{abstract}
We present a study of vortex formation in interface biased nano-sized disk and square nanoelements of $\mathrm{Fe}$ and $\mathrm{Py}^{\mathrm{TM}}$. For small lateral dimensions, the circular nanoelements have smaller vortex core diameter than square nanoelements with equal top surface area. For surface area ranging from $1900 \mathrm{~nm}^{2}$ to $6700 \mathrm{~nm}^{2}$, the vortex core diameter of $30 \mathrm{~nm}$ thick Fe $\left(\mathrm{Py}^{\mathrm{TM}}\right)$ nanoelements varies from $32 \mathrm{~nm}(36 \mathrm{~nm})$ to $36 \mathrm{~nm}(48 \mathrm{~nm})$. Interface effects are stronger for Py ${ }^{\mathrm{TM}}$ nanoelements. (C) 2012 American Institute of Physics. [doi:10.1063/1.3675989]
\end{abstract}

\section{INTRODUCTION}

The magnetic profile of the vortex core in confined magnetic nanoelements is relevant for a number of phenomena of current interest. Tailoring the vortex core is a key issue for applications based on vortex core dynamics, ${ }^{1}$ such as spin-transfer torque micro-wave nano-oscillators, ${ }^{2,3}$ currentdriven vortex oscillations in metallic nanocontacts, ${ }^{4}$ phase locking of the vortex dynamics in nano-oscillator arrays, ${ }^{5}$ and controlling the vortex interaction in layered nanostructures. $^{6}$

Confinement may lead to considerable changes in the shape and size of the vortex core, as well as on the vortex core surroundings. ${ }^{7}$ The static vortex profile may deviate strongly from an ideal vortex profile, ${ }^{8}$ where the magnetization lies in-plane everywhere but in the vortex core.

Dipolar effects are strongly shape dependent. Thus confinement effects on the vortex core of square basis nanoelements are likely to be different from those on circular nanoelements.

Furthermore, by exchange coupling a ferromagnetic nanoelement to a large uniaxial anisotropy antiferromagnetic substrate, one favors the formation interface areas with spins locked to the interface field direction. ${ }^{9-11}$ Thus, even for small height nanoelements, interface biasing may lead to a layer dependent magnetic profile.

In this paper, we report a theoretical study of the static equilibrium structure of vortices in small area $\mathrm{Fe}$ and $\mathrm{Py}^{\mathrm{TM}}$ nanoelements. We consider $30 \mathrm{~nm}$ thick nanoelements with top surface area ranging from $1936 \mathrm{~nm}^{2}$ to $6724 \mathrm{~nm}^{2}$.

The point of choosing flat nanoelements is that one may explore the two elements, confinement and interface biasing, in a clear manner. Unbiased $30 \mathrm{~nm}$ thick nanoelements of the lateral dimensions chosen in this study form vortex states with the same pattern from the interface up to the free surface layer.

We show that the impact of the lateral dimensions on the overall arrangement of spins to minimize the stray field energy is larger for circular nanoelements. For nanoelements

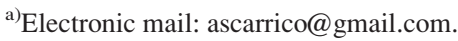

with the same surface area and height, the vortex core radius of a nanodisk is smaller than that of a square nanoelement. The difference turns out negligible for dimensions much larger than exchange length (see Table I). Also, the vortex state of $\mathrm{Py}^{\mathrm{TM}}$ nanoelements is more susceptible to interface biasing.

The normal to the surface is chosen along the $\mathrm{z}$-axis and the uniaxial anisotropy easy axis and the interface field are both in-plane and along the $\mathrm{x}$-axis. The antiferromagnetic substrate is held frozen in the antiferromagnetic order. We use cubic simulation cells with edge $d=2 \mathrm{~nm}$ and the energy density is given by

$$
\begin{aligned}
E= & \frac{A}{d^{2}} \sum_{j} \sum_{k}\left(1-\hat{m}_{j} \cdot \hat{m}_{k}\right)-M_{S} \vec{H}_{\mathrm{int}} \cdot \sum_{i} \hat{m}_{i} \\
& -M_{S} \vec{H} \cdot \sum_{j} \hat{m}_{j}-K \sum_{j}\left(m_{j}^{x}\right)^{2} \\
& +\frac{M_{S}^{2}}{2} \sum_{j} \sum_{k}\left(\frac{\hat{m}_{j} \cdot \hat{m}_{k}}{n_{j k}^{3}}-\frac{3\left(\hat{m}_{j} \cdot \hat{n}_{j k}\right)\left(\hat{m}_{k} \cdot \hat{n}_{j k}\right)}{n_{j k}^{5}}\right),
\end{aligned}
$$

where the first term is the intrinsic exchange energy, coupling magnetic moments of nearest-neighbor cells and $A$ is the ferromagnetic exchange stiffness. The second term is the interface exchange energy, and the sum is restricted to the first layer of cells, at the interface. The third and fourth terms are the Zeeman and anisotropy energies, and the last

TABLE I. Vortex core diameter (nm).

\begin{tabular}{lcccccc}
\hline \hline & & & \multicolumn{3}{c}{ Circular } \\
\cline { 5 - 7 } Edge $a(\mathrm{~nm})$ & $\mathrm{Fe}$ & $\mathrm{Py}^{\mathrm{TM}}$ & & Radius $r(\mathrm{~nm})$ & $\mathrm{Fe}$ & $\mathrm{Py}^{\mathrm{TM}}$ \\
\hline 44 & - & 44 & & 21 & 32 & 36 \\
46 & - & 44 & & 23 & 32 & 44 \\
48 & 36 & 44 & 25 & 32 & 44 \\
50 & 36 & 44 & 27 & 32 & 44 \\
52 & 36 & 46 & & 29 & 36 & 44 \\
54 & 36 & 46 & & 31 & 36 & 44 \\
82 & 36 & 48 & & & 36 & 48 \\
\hline \hline
\end{tabular}




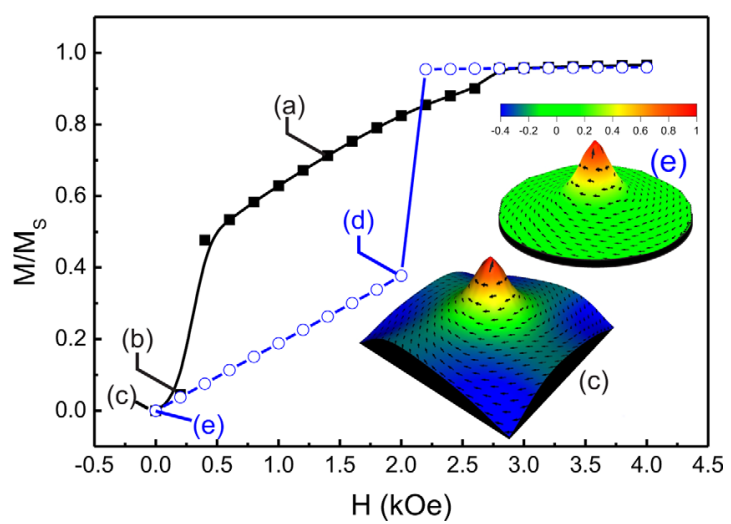

FIG. 1. (Color online) Magnetization of $30 \mathrm{~nm}$ thick Fe nanoelements: (a) square with lateral dimensions $48 \mathrm{~nm} \times 48 \mathrm{~nm}$ and (b) circular with diameter of $54 \mathrm{~nm}$. The panels show the magnetic structure of the vortex cores, and the color bar code show the z-component of the magnetic moment.

term is the magnetostatic energy. $M_{S}$ is the saturation magnetization, $\hat{m}_{i}$ is the direction of the magnetic moment of the $i$ th cell, and $n_{i j}$ is the distance between the cells $i$ and $j$ in units of cell size $d$.

For iron, we use $M_{S}=1.7 \times 10^{6} \mathrm{~A} / \mathrm{m}, A=2.5 \times 10^{-11} \mathrm{~J} / \mathrm{m}$, and $K=4.7 \times 10^{-4} \mathrm{~J} / \mathrm{m}^{3}$. For Permalloy ${ }^{\mathrm{TM}}$, we use $M_{S}=$ $0.8 \times 10^{6} \mathrm{~A} / \mathrm{m}, A=1.3 \times 10^{-11} \mathrm{~J} / \mathrm{m}$, and $K=0$.

For each value of the external field strength, the equilibrium configuration is found by seeking a set of directions of the moments in all cells $\left(\hat{m}_{i}, i=1, \ldots, N\right)$, which makes the torque in each one of them smaller than $10^{-26} J_{.}{ }^{10,11}$

We obtain the equilibrium pattern at remanence by starting from saturation at large external field values $(H)$ along the $\mathrm{x}$-axis direction (4 kOe was enough to achieve saturation for all nanoelements). Then, we followed the sequence of equilibrium states using a fine grid of values of $H$, all the way down to $H=0$ (see Fig. 1). The equilibrium profile for each value of the external field strength was used as the initialization pattern for the next value of the external field strength.

The differences in the magnetic structure of the vortex core of small area square and circular nanoelements originate in the adjustment of surface and volume charges near the lateral surfaces. As shown in Fig. 1 (inset panels) and Fig. 2, near the corners of square nanoelements, there are stronger changes of the perpendicular component of the local magnetic field. Circular nanoelements have a smaller variation of the local field near the lateral surfaces and larger areas with in-plane moments around the vortex core.

Also, the square nanoelements have magnetic phases with no counterpart for the circular nanoelements. Typical results are shown in Figs. 1 and 2 for Fe nanoelements of nearly the same top surface area. We consider a $30 \mathrm{~nm}$ thick square Fe nanoelement with edge of $48 \mathrm{~nm}$ and a circular nanoelement of same height and diameter of $54 \mathrm{~nm}$.

The nucleation of non-uniform states in the square nanoelement involves the formation of a twisted state, ${ }^{10}$ prior to the formation of the vortex state. The twisted state consists in a magnetic pattern in which each layer is almost saturated (in a s-state), and there is a turn angle between the direction of the average magnetization of successive layers. The relative orientation of the average magnetization of the top and bottom layers evolves from nearly parallel, near saturation, to almost perpendicular at small values of $H$. At low external field, values an off-center vortex state is formed and evolves to the vortex state in the center of the nanoelement at remanence.

The uniform state of the circular nanoelement is stable from saturation down to a field of $2 \mathrm{kOe}$. At this point, an off-center vortex is formed. The vortex core shifts
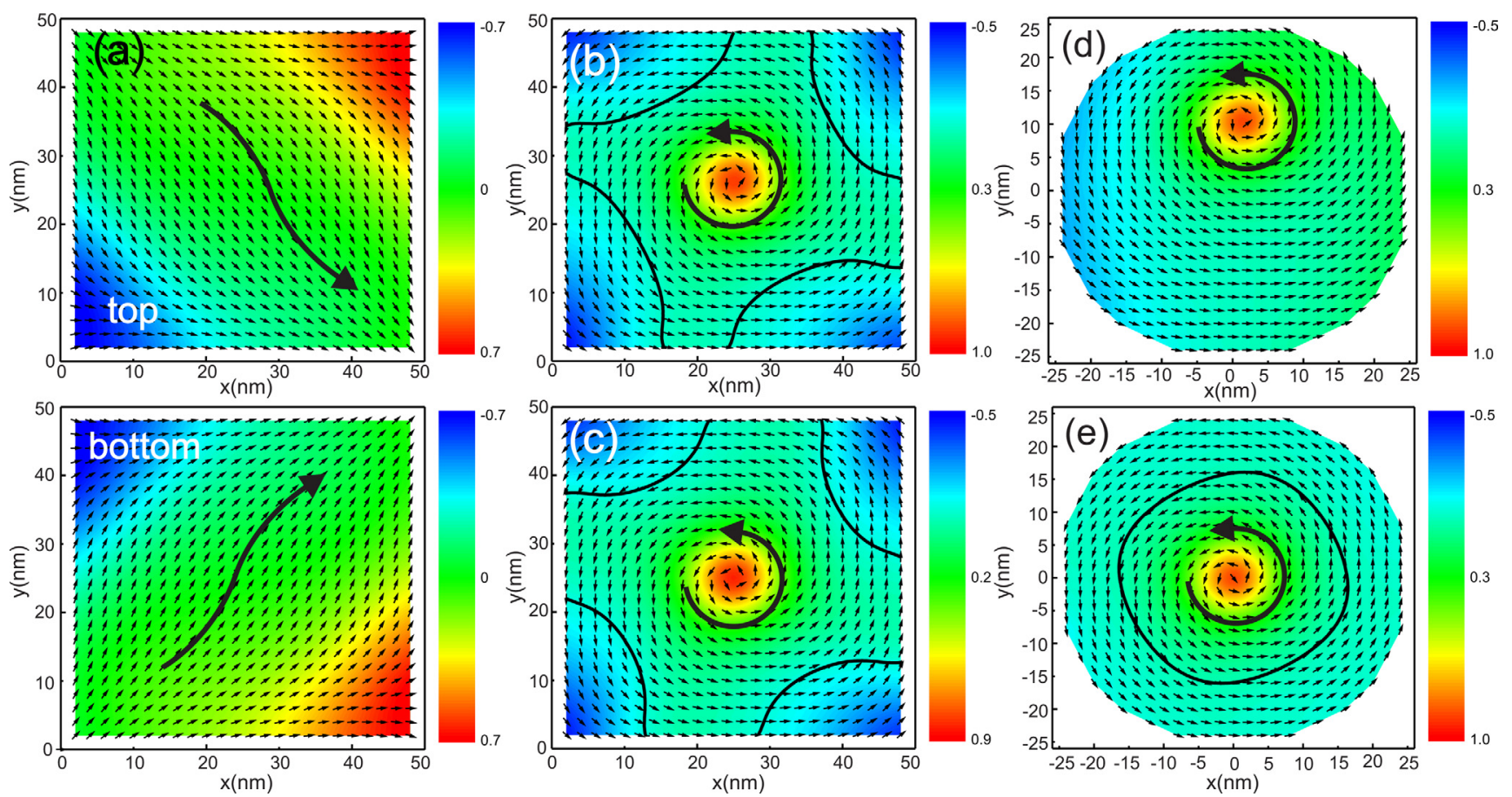

FIG. 2. (Color online) Magnetic structure at selected points of the magnetization curves of Fig. 1. The bar codes show the z-component the magnetization, and the continuous lines, in panels (b), (c), and (e), separates areas with opposite sign of the z-component of the local field. 

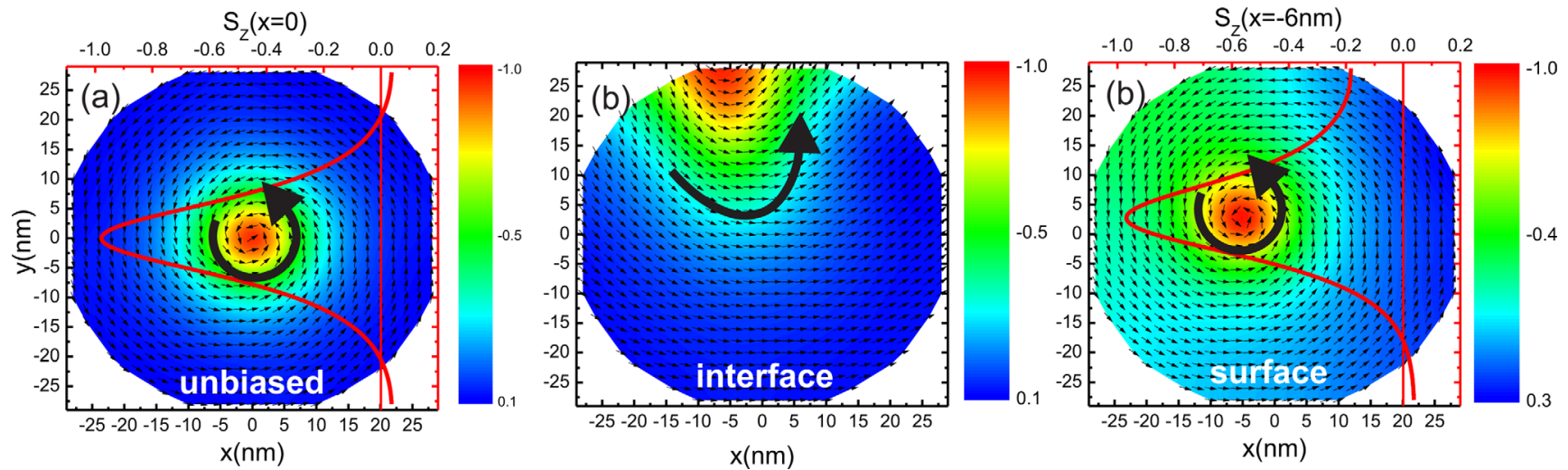

FIG. 3. (Color online) Magnetic structure of the vortex core of (a) unbiased and (b) interface biased $30 \mathrm{~nm}$ thick circular Py ${ }^{\mathrm{TM}}$ nanoelement with $62 \mathrm{~nm}$ diameter. The color bar code shows the z-component $\left(S_{z}\right)$ of the magnetization and the continuous lines show $S_{z}$ in the vortex core at (a) $\mathrm{x}=0$ and (b) $\mathrm{x}=-6 \mathrm{~nm}$, at the surface layer.
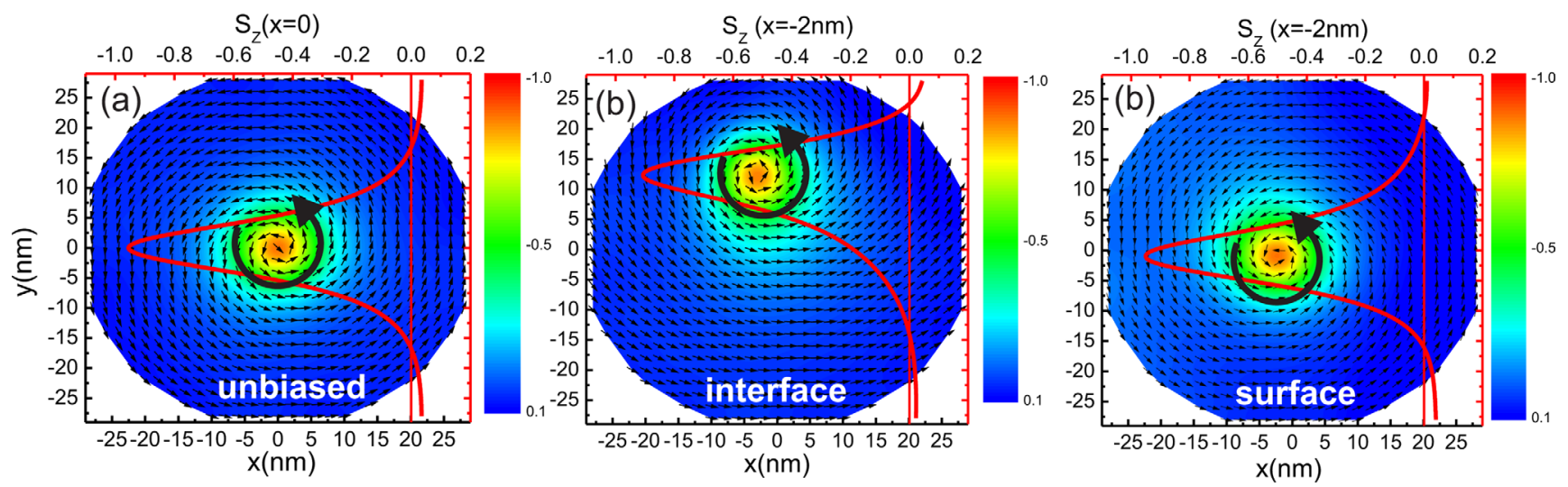

FIG. 4. (Color online) Magnetic structure of the vortex core of (a) unbiased, (b) interface layer, and (c) surface layer of interface biased $30 \mathrm{~nm}$ thick circular Fe nanoelement with $62 \mathrm{~nm}$ diameter. The color bar code shows the z-component $\left(S_{z}\right)$ of the magnetization and the continuous lines show $S_{z}$ in the vortex core at (a) $\mathrm{x}=0,(\mathrm{~b}) \mathrm{x}=-2 \mathrm{~nm}$ at the interface layer, and (c) $\mathrm{x}=-2 \mathrm{~nm}$ at the surface layer.

continuously toward the center of the nanoelement as the external field is reduced to $H=0$.

The overall impact of dipolar energy differences results in larger variation of the diameter of the vortex core for circular nanoelements, as shown in Table I. The threshold value of the top surface for formation of a vortex is smaller for circular nanoelements. The same phenomenology applies for $\mathrm{Py}^{\mathrm{TM}}$ nanoelements for slightly larger values of the diameter of the vortex core.

$\mathrm{Py}^{\mathrm{TM}}$ and $\mathrm{Fe}$ nanoelements differ considerable in one more key point. Interface bias affects more strongly the magnetic structure of $\mathrm{Py}^{\mathrm{TM}}$ nanoelements.

Figures 3 and 4 display the interface bias changes of the vortex state of $30 \mathrm{~nm}$ thick circular nanoelements with a diameter of $62 \mathrm{~nm}$, for an interface field strength of $10 \mathrm{kOe}$. There is a larger impact on the magnetic profile in the $\mathrm{Py}^{\mathrm{TM}}$ nanoelement. As shown in Fig. 3, the unbiased nanoelement has a vortex core, with diameter of $44 \mathrm{~nm}$, located at the nanoelement center. The interface biased structure is off-center, from the bottom (interface) layer up to the surface layer. Furthermore, due to change in position of the core from layer to layer, the normal component of the magnetization is nonzero across the whole diameter of the nanoelement.

For $\mathrm{Fe}$, the dipolar field is stronger, and the vortex state is more stable. Interface biasing leads to smaller changes. As shown in Fig. 4, the changes of the unbiased vortex pattern are smaller. However, there is a shift of core position in the interface layer, leading to a larger area with the interface spins aligned with the interface field.

We note that the interface bias control of inhomogeneities of the magnetic structure of the vortex might be of interest in the discussion of the vortex oscillations driven by spin polarized steady currents.

\section{ACKNOWLEDGMENTS}

The authors acknowledge financial support from FAPERN, CNPq, and CAPES.

${ }^{1}$ S.-B. Choe, Y. Acremann, A. Scholl, A. Bauer, A. Doran, J. Stöhr, and H. A. Padmore, Science 304, 420 (2004).

${ }^{2}$ A. Dussaux et al., Nature Commun. 1, 1006 (2010).

${ }^{3}$ A. Dussaux et al., Appl. Phys. Lett. 98, 132506 (2011).

${ }^{4}$ M. van Kampen et al., J. Phys. D: Appl. Phys. 42, 245001 (2009).

${ }^{5}$ S. Kaka et al., Nature 437, 389 (2005).

${ }^{6}$ K. Yu. Guslienko, K. S. Buchanan, S. D. Bader, and V. Novosad, Appl. Phys. Lett. 86, 223112 (2005).

${ }^{7}$ X. W. Yu et al., Phys. Rev. Lett. 106, 167202 (2011).

${ }^{8}$ T. Shinjo, T. Okuno, R. Hassdorf, K. Shigeto, and T. Ono, Science 289, 930 (2000).

${ }^{9}$ A. S. M. Silva, A. L. Dantas, G. O. G. Rebouças, and A. S. Carriço, J. Appl. Phys. 109, 07D314 (2011).

${ }^{10}$ G. O. G. Rebouças, A. S. W. T. Silva, A. L. Dantas, R. E. Camley, and A. S. Carriço, Phys. Rev. B 79, 104402 (2009).

${ }^{11}$ A. L. Dantas, G. O. G. Rebouças, A. S. W. T. Silva, and A. S. Carriço, J. Appl. Phys. 97, 10K105 (2005). 